\title{
Hot-cathode Preionization Studies in CCT
}

\author{
D. S. Darrow and M. Ono \\ Princeton University, Plasma Physics LaUoratory \\ P.O. Box 451 \\ Princeton, NJ 08543 \\ P. A. Pribyl and R. J. Taylor \\ Tokamak Fusion Laboratory \\ 1540 Boelter Hall \\ University of California at Los Angeles \\ Los Angeles, CA 90024
}

\begin{abstract}
A hot $\mathrm{LaB}_{6}$ cathode was used to ionized the gas in the vessel of CCT at the start of tokamak discharges. Substantial plasma densities could be obtained in the preionization phase, resulting in reliable breakdown and initiation of $g_{a} \simeq 3$ discharges at loop voltages of $4.2 \mathrm{~V} / \mathrm{turn}$, considerably lower than the $33 \mathrm{~V} /$ turn required with no preionization and the $20 \mathrm{~V} /$ turn required when a $15 \mathrm{kHz}$ oscillator was the preionization source. When inductive effects were subtracted, the cathode preionization produced a loop voltage at tributable to plasma resistance of $4 \mathrm{~V} /$ turn, while the oscillator-produced plasma required $12 \mathrm{~V} /$ turn. Repeatable 1.2thode-enhanced breakdowns could be obtained at voltages as low as $3.4 \mathrm{~V} /$ urn for discharges with higher $q_{a}$. With the cathodeenhanced plasma, the initial value of $d I_{p} / d \Phi_{O H}$ is higher than that with the oscillator-produced plasma. The spectrum of visible light emitted from cathode-initiated discharges shows no additional impuritich present beyond those seen in a normal plasma.
\end{abstract}




\section{Introduction}

Recent years have scen proposals for and planning of several large new tokamaks, such as the International Thermonuclear Experimental Reactor ${ }^{1}$ and the Burning Plasma Experiment. ${ }^{2}$ Designs for these devices, as well as for several smaller ones, call for toroidally continuous vacuum vessels of substantial thickness for the purpose of preventing distortion of the vessel during disruptions. For instance, the vessel of BPX is planned to be $10 \mathrm{~cm}$

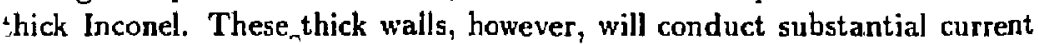
when the toroidal electric field is applied to drive plasma current, and this effect will be most severe at the start of the discharge when the loop voltage is highest. Current flow in the vacuum vessel will increase the fraction of energy from the ohm z heating system that is lost (i.e. does not appear as magnetic energy in the plasma).

In order to prevent this waste of ohmic heating energy, some prejonization of the wisking gas will almost certainly be performed before the loop voltage is applied. There are numerous means of providing preionization, including the use of a Penning trap with a short connection to the tokamak vessel, placement of a hot tungsten filament in the vessel, and application of $\mathrm{rf}$ power to the gas (including electron-cyclotron resonance radiation). Of these, ECH preionization is probably most well studied. ${ }^{3-6}$ This technique was shown to reduce the amount of flux and energy consumed in the breakdown phase of the discharge in studies performed on CLEO. Other beneficial properties were demonstrated on ISX-B and Tokapole II. The purpose of this paper is to report results from a new preionization technique, namely a biased hot cathode. Section II describes the experimental apparatus and procedure, Section Ill describes the results obtained, and Section IV describes possible implementaitions of the scheme, as well as areas where more experimentation might be fruit.ful.

\section{Apparatus and Procedure}

The objective of cathode preionization is to produce as large an elentron density and temperature as is possible over as large a volume of the vacuum vessel as is possible. This is achieved by collisional ionization and heating of the working gas by electrons emitted by the cathode. In order for the 
cathode to emit injtially, the distance along a magnetic field line between the cathode and anode (the vacuum vessel and liner in this case) must not exceed a a few times the mean free path of electrons in the working gas. (If this condition is not met, the resistance of the working gas to electron flow will limit the amount of power that can be deposited, significantly reducing the amount of ionization produced.) The simplest means of obtaining the desired configuration is to apply some vertical field (in addition to the toroidal field) when the preionization is to take place. However, once sufficient plasma density is created and the toroidal electric field is applied, vertical field becomes detrimental, as it allows current-carrying electrons from the plasma to escape along field lines and strike the walls. Therefore, as small a vertical field is possible should be used. If even this field is too large, then some time must be allowed to elapse between the end of the preionization and the application of the toroidal electric field. In this time, the vertical field mist decay to an acceptable value. Fortunately, in the experiment described herc, suffciently little vertical field was required that the toroidal electric fieid could be applied before the plasma density fell by more than $20 \%$.

Figure 1 depicts the experimental arrangement used to try this preionization technique on the Continuous Current Tokamak (CCT) at UCLA. CCT has $R=1.5 \mathrm{~m}, a=0.4 \mathrm{~m}$, and was operated with $B_{T}=0.3 \mathrm{~T}, I_{F}=40 \mathrm{kA}$, and $\bar{n}_{e}=1-3 \times 10^{18} \mathrm{~m}^{-3}$. During cathode preionization experiments, the cathode was inserted $0.1 \mathrm{~m}$ beyond the edge of the vessel liner and limiter. This effectively reduced the minor radius to $a=0.35 \mathrm{~m}$.

In the experiment on CCT, the cathode was located at the top of the plasma cross-section, at a single toroidal location. The cathode constsuction is described elsewhere ${ }^{7-9}$ and consists of a set of cylindrical beads- of lanthanum hexaboride $\left(\mathrm{LaB}_{6}\right)$ strung onto a carbon rod which is electrically heated. The whole assembly may be biased with respect to the vacuum vessel and liner, these latter serving as the anode. Electrons emitted by the cathode flow preferentially along the direction of the magnetic field. A tungsten plate on one side of the $\mathrm{LaB}_{6}$ assembly is held at the cathode potential, effectively blocking electron emission on that side. As a consequence, electrons are emitted only on the opposite side where, because of the field geometry, they are forced to cross the volume of the torus betore striking the liner at the bottom of the machine. The cathode was originally installed in CCT for the purpose of testing dc heli ity injection as a means of tokamak current 
drive. ${ }^{8}$ The cathode was used without modification for this experiment.

The CCT vacuum vesse] is fabricated from $1 \mathrm{~cm}$ thick stainless steel, and it has an insulating break to interrupt the flow of current in the toroidal direction. The vessel liner is comprised of copper panels, $3 \mathrm{~mm}$ thick, which are bolted to the vacuum vessel. The liner is broken in numerous places both toroidally and poloidally. Loop voltzge is measured as the voltage developed across the insulating break in the vessel and, in the absence of the hot cathode preionization, CCT discharges are initiated by a breakdown oscillator which applies a $15 \mathrm{kHz}, 100 \mathrm{~V}$ sine wave arross the insulating break. This preionization method produces a uniform plasma with $n_{\mathrm{e}} \sim 10^{17} \mathrm{~m}^{-3}$.

A typical discharge sequence starts with the application of voltages to the toroidal field and ohmic heating coils. Once these approach their maximum currents, either the breakdown oscillator or the cathode voltage is applied. Shortly thereafter, a small capacitor bank is discharged into the vertical field coil. The purpose of this pulse is (a) to create a fieid null in the vessel in the case of ordinary breakdown, or (b) to supply the vertical field required for proper plasma production by the cathode. Shortly after this pulse is applied, the toroidal voltage is applied by commutating the current in the ohmic heating winding. At the same time, the vertical field used to maintain plasma equilibrium is turned on.

In the experiment, it was observed that current would flow from the cathode under some circumstances before the vertical field prepulse was applied. This, it is thought, was due to stray fields produced by eddy currents in the vacuum vessel. Since these fields resulted in the ability to create plasma and draw current from the cathode, no effort was made to eliminate them. Obviously, all forms of startup are sensitive to such stray fields, and detaited knowledge of their origins and magnitudes can only assist in refining the breakdown conditions. As this experiment was of an initial nature, no such detailed knowledge was sought.

\section{Results}

Figure 2 shows representative data from two shots: (a) tlirough (g) with cathode preionization, and (h) through (n) with breakdown oscillator preionization. (Technical limitations allow only the first $100 \mathrm{msec}$ of each discharge to be recorded, hence the full time evolution of these discharges is not de- 
picted.) All discharges discussed herein were formed in hydrogen. For the cathode-initiated case, a bias voltage of about $750 \mathrm{~V}$ was applied, resulting in a peak injected current of 320 A. Injected power from the cathode peaked at $180 \mathrm{~kW}$, and exceeded $150 \mathrm{~kW}$ for 5 msec. The toroidal electric field was first applied after 4 msec of this high-power injection.

The jnitial filling pressure of hydrogen was $6 \times 10^{-5}$ Torr (gauge). During cathode preionization, the ultravjolet emission (see below and Fig. 4) indicated that plasma was forming in the uppermost $20 \mathrm{~cm}$ of the vessel. The highest line-averaged density, as measured by a $4 \mathrm{~mm}$ interferometer viewing a vertical chord passing through the vessel centerline, was $\bar{n}_{e}=1.4 \times 10^{19} \mathrm{~m}^{-3}$ during cathode biasing. During the main, uhmic-driven portion of the discharge, the interferometer indicates a density of $\bar{n}_{e}=2 \times 10^{38} \mathrm{~m}^{-3}$, and the UV detector data indicate that the plasma fills the vessel.

The ultraviolet delector array data for cathode-initiated and oscillatorinitiated discharges are shown in Figs. $4(\mathrm{a})$ and $4(\mathrm{~b})$. The array consists of 16 detectors, eight which view the plasma along vertical chords, and eight which view the plasma along horizontal chords. In both views, the detectors are spaced at $\pm 0.05 \mathrm{~m}, \pm 0.15 \mathrm{~m}, \pm 0.25 \mathrm{~m}$, and $\pm 0.35 \mathrm{~m}$ from the vessel centerline. (In the data presented in Fig. 4, however, the horizontal chord detector at $+0.25 \mathrm{~m}$ has been omitted due to an instrumental malfunction which produced an erroneous signal for the shots of interest.) The signal from the UV detectors is assumed to be predominantly $H_{\alpha}$ radiation. Before these measurements were made, the gains on all 16 channels of the UV detector array were adjusted so that all produced an equal amplitude signal when viewing a hydrogen discharge cleaning plasma in the CCT vessel. Previous observations ${ }^{10}$ have indicated that discharge cleaning plasmas have volumetrically uniform light emission. This calibration then means that the detector outputs are proportional to the line-average UV emissivity along the chords viewed. The vertical chord data from the cathode breakdown case show the formation of a plasma with a full width at hall maximum of $\sim 30 \mathrm{~cm}$; the horizontal chord data indicate that UV emission is localized to the uppermost $20 \mathrm{~cm}$ of the chamber, as noted above. As the breakdown proceeds, the peak UV emission is seen to shift from the center of the vessel to the outboard edge. It subsides briefly, then rises again for a few milliseconds at the outside edge after the toroidal electric field is appliad. This latter signature suggests that the plasma is scraping the outboard section of the liner for the first few 
milliseconds, which may account for the short interruption of the current rise then. In the oscillator-initiated plasma, a peak in UV emission is seen when the $15 \mathrm{kHz}$ voltage is applied; the emission is nearly uniform spatially. In both types of discharges, the UV emission is slightly centrally-peaked and small once the discharge is fully established.

At an absolute minimum, oscillator-iaitiated discharges would not break down below $20 \mathrm{~V} /$ turn, and discharges with no preionization at all failed below $33 \mathrm{~V} /$ turn. In the oscillator-initiated discharge depicted in Fig. 2, the peak loop voltage is $34 \mathrm{~V}$, dropping to $2.5 \mathrm{~V}$ at the current flat-top. In contrast, the cathode-initiated discharge at no time has a loop voltage higher than $4.2 \mathrm{~V}$; the loop voltage drops to $2.2 \mathrm{~V}$ as the current reaches its peak value. Cathode-preionized discharges could be formed with loop voltages as low as $3.4 \mathrm{~V}$. Under the latter conditions, however, the peak plasma current was reduced by a factor of two. If the normalized internal inductance of the plasma is assumed to be $\ell_{i}=1$ (there is no diagnostic of this quantity on CCT), then the total piasma inductance, assuming constant major and minor radii throughout the shot, is approximately $L_{\text {plamn }}=3.8 \mu \mathrm{H}$. The portion of the applied loop voltage which is increasing the plasma current against its inductance is $L_{\text {plasma }} \frac{d \underline{l}_{l}}{d t}$. Subtraction of this quantity from the measured loop voltage provides an estimate of the voltage drop around the plasma circuit due solely to the plasma resistance. This fraction is plotted in Figs. 2(g) and 2(n). At breakdown in the oscillator-initiated discharge, this value is $12 \mathrm{~V} / \mathrm{turn}$, while in the cathode-initiated discharge it never exceeds $4.5 \mathrm{~V} /$ turn, representing a significant improvement in plasma conductivity.

Figure 4(a) shows the plasma current plotted against ohmic heating primary current $\left(I_{O H}\right)$ for the two types of discharges. The ohmic heating coil current is linearly related to the ohmic heating flux, and so this figure displays the efficiency of $\mathrm{OH}$ flux usage in the two types of discharges. Note that just after breakdown, the cathode-injtiated discharge exhibits a larger $d I_{p} / d I_{O H}$ than does the oscillator-initiated discharge. After a brief interval of rapid rise, the current remains virtually constant for a few milliseconds before resuming its increase. The increase of UV emissions at the outboard wall suggest that, at this point, the plasma is scraping against the wall. This suggests that better control of the plasma position would allow the period of enhanced $d I_{p} / d I_{O H}$ to persist longer. Once the discharges are well-established, however, the traces possess (and are expected to possess) very similar slopes. 
Some of the discharges display the same current waveform, but lack the UV emissions at the outboard wall, limiting the confidence placed in the above interpretation of the delay in the current rise. Alternatively, the delay may be a result of the loss of significant input power from the electron beam as the magnetic configuration of the plasma changes and as it moves away from the cathode.

The issue of energy consumption during breakdown and startup of these discharges is difficult io assess given the differing rates of current rise. Figure 4 (b) displays the plasma current attained versus the energy expended for the two discharges considered previously, where the energy input to each discharge is computed as the time-integral of the quantity $V_{\text {cain }} I_{\text {inj }}+V_{\text {loop }} I_{p}$. The traces in this figure are arranged so that zero energy coincides with the point at which the ohmic heating is applied, i.e. energy input shown to the left of zero is from the preionization source, while that to the right of zero is due chiefly to the ohmic heating system. In the cathode case, $750 \mathrm{~J}$ is applied during preionization, and $450 \mathrm{~J}$ is opplied by the cathode after the loop voltage is applied, while in the $15 \mathrm{kHz}$ oscillator case, preionization is estimated to supply approximately $100 \mathrm{~J}$ to the plasma, all before the loop voltage is applied. The highest value of incremental efficiency, $d I_{p} / d E_{t o c}$ is found during the first few milliseconds of the oscillator-initiated plasma, and is $\sim 200 \mathrm{~A} / \mathrm{J}$. In contrast, the incremental efficiency in the cathode-initiated discharge never exceeds $40 \mathrm{~A} / \mathrm{J}$. The cathode-initiated discharge, however, maintains a higher incremental efficiency over a longer period in order eventually to exceed the current in the oscillator-initiated discharge. The reasons for the observed eficiencies are not clear at present, and presumably are influenced by the magnetic configuration of the discharge, the rate of burnthrough of low- $Z$ impurities, as well as the initial degree of ionization.

One additional fact worthy of mention is that measurements of visible spectral lines in the $420 \mathrm{~nm}$ to $490 \mathrm{~nm}$ range revealed no additional plasma impurities when the cathode was present. In particular, no evidence of boron lines (from the $\mathrm{LaB}_{6}$ ) was seen, and no enhancement of the carbon lines in the spectra was seen (there are carbon components in the cathode, and the CCT device has only very small amounts of graphite exposed to the plasma). Hence, at these volumetric power levels, the hot cathode provides a clean method of discharge formation. 


\section{Possible Applications of the Technique}

Prcionization could provide a significant advantage to tokamaks with toroidally-continuous vessels in that it can increase the ability to breakdown at low values of the toroidal electric field. Lower toroidal electric fields would mean lower losses to eddy currents in the vessel, and smaller stray fields. Cathode preionization on a large device could proceed in essentially the same fashion used on CCT, although a greater degree of control of the vertical and poloidal fields would be of value.

Cathode preionization has the advantage that it is simple, low-cost, efficient in its production of plasma, and reliable. In comparison to ECH breakdown, it is not limited in the maximum density it can create (which is the density at which $\omega_{p c}=\Omega_{c}$, or $9 \times 10^{17} \mathrm{~m}^{-3}$ for CCT). In addition, the cathode is able to create plasma over a broader range of filling pressure than is the ohmic system alone. This may have some implications for discharge control. To its detriment, cathode preionization does rely upon in-vessel components. These require more complex replacement procedures than would an ECH source located outside a radiation wall. Also, the cathode discharge relies upon the applied vertical field to be able to for u a plasma at all. Machines with thick vacuum vessels may not allow that vertical field to decay suficiently rapidly to allow normal breakdown once the cathode has created a plasma.

Future research on this topic could pursue a number of different issues. Among them are these: Can the vertical field be controlled sufficiently to utilize the techinique in vessels with thick walls? What refinements to the method are required in devices with higher toroidal fields? To what extent can energy supplied by the cathode replace energy supplied by the ohmic heating system?

\section{Summary}

Preionization of a tokamak discharge was performed in the CCT device with an emissive $\mathrm{LaB}_{6}$ cathode, and has been compared to initiation of discharges with a $15 \mathrm{kHz}$ breakdown oscillator. The cathode produced plasmas with densities locally of up to $1.4 \times 10^{19} \mathrm{~m}^{-3}$, a factor of 100 denser than oscillator-produced plasmas, and five times more dense than the ohmic dis- 
charges in CCT. UV emissions indicate that the cathode plasma is concentrated in the top $20 \mathrm{~cm}$ of the vacuum vessel, while oscillator discharges, and the main tokamak discharges, occupy a much larger percentage of the vessel. Cathode preionization allowed the tokamak discharge to be started with loop voltages as low as $3.4 \mathrm{~V}$ for derated discharges, and as low as $4.2 \mathrm{~V}$ for normal $q_{a}=3$ discharges. This compares favorably with a $20 \mathrm{~V}$ minimum required using the breakdown oscillator and a $33 \mathrm{~V}$ minimum in the absence of any preionization. After subtraction of the $L_{\text {plama }} \frac{d I_{F}}{d t}$ contribution to the loop voltage, the remaining voltage was $\sim 12 \mathrm{~V}$ in the oscillator-initiated discharge and $\sim 4 \mathrm{~V}$ in the cathode-initiated discharge. With the cathode, $d I_{p} / d \Phi_{o H}$ was initially larger than with the cscillator, meaning ohmic heating flux was being used more efficiently. Cathode preionization may have some benefits for tokamaks whose vacumm vessels will be toroidally continuous and of low resistance.

The authors wish to acknowledge useful discussions with Dr. M. Bell of Princeton Plasma Physics Laboratory, and the contributions of M. Yurchansky, C. Wannberg, and G. Miskell, who provided able technical assistance. This work was supported by U.S. Department of Energy Contracts DE-ACO2-76-CHO-3073 and DE-FG03-86ER53225. 


\section{References}

${ }^{\prime} \mathrm{R}$. Toschi, in Proceedings of the Thirteenth International Confenence on Plasma Physics and Controlled Nuclear Fusion, (IAEA, Vienna. 1991).

${ }^{2}$ D. J. Sigmar, et al, in Proceedings of the Thirteenth International Conference on Plasma Physics and Controlled Nuclear Fusion, (IAEA, Vienna, 1991).

${ }^{3}$ B. Lloyd and T. Edlington, Plasma Phys. Contr. Fusion 28, 909-915 (1986).

${ }^{4}$ A. Kulchar, O. C. Eldridge, A. C. England, et al., Phys. Fluids 27, 1869 1879 (1984).

${ }^{5}$ D. J. Holly, S. C. Prager, D. A. Shepard, and J. C. Sprott, Nucl. Fusion 21, 1483-1487 (1981).

${ }^{6}$ A. Pochelon, T. Goodman, D. Whaley, et al., to appear in Proceedings of the I6th Symposium on Fusion Technoloyy, London, 3-7 September 1990.

${ }^{7}$ M. Ono, G. J. Greene, D. Darrow, C. Forest, H. Park, and T. H. Stix, Phys. Rev. Lett. 59, 2165-2168 (1987).

${ }^{8}$ D. S. Darrow, M. Ono, C. B. Forest, et al., Phys. Fluids B 2, 1415-1420 (1990).

${ }^{9}$ D. S. Darrow, Ph.D Thesis, Princeton Unjversity, 1988.

${ }^{10} \mathrm{~J}$. R. I.jberati, private communication, based upon observaíions of visjble light emissions from cleaning discharges in the Microtor vessel. 
Figure 1 Experimental configuration on CCT. The ressel minor radius is $0.5 \mathrm{~m}$, that of the liner is $0.4 \mathrm{~m}$. The cathode, when it is used, projects $0.1 \mathrm{~m}$ inward from the liner, at the top of the torus. The loop voltages presented in this article those rneasured across the insulating gap in the vessel. The usual means of prejonization in CCT is a $15 \mathrm{kJ/z}$ oscillator which applies a $100 \mathrm{~V}$ sine wave to the gap in the vessel.

Figure 2 The time-histories of a cathode-initiated discharge and an oscillatorinitiated discharge. (a) Loop voltage, cathode discharge. (b) Plasma current, cathode discharge. (c) Vertical field current, cathode discharge. (d) Cathode voltage, cathode discharge. (e) Cathode current, cathode discharge. (f) Ohmic input power (solid line) and cathode input power (dashed line), cathode discharge. (g) $V_{L}-L_{\text {plamma }} \frac{d I_{z}}{d t}$, cathode discharge. (h) Loop voltage, oscillator discharge. (i) Plasma current, oscillator discharge. (j) Vertical field current, oscillator discharge. (k) Cathode voltage, oscillator discharge. (I) Cathode current, oscillator discharge. (m) Total input power (cathode+ohmic), oscillator discharge. (n) $V_{L}-L_{\text {plasma }} \frac{d h_{2}}{d t}$, oscillator discharge. (Note the differences in scales between $(\mathrm{a})$ and $(\mathrm{h}),(\mathrm{f})$ and $(\mathrm{m})$, and $(\mathrm{g})$ and (m).)

Figure 3 (a) The UV emission seen along vertical chords through the vessei, from a cathode-initiated plasma, before, during, and after preionization. (b) UV emission in the same plasma, seen along horizontal chords. (c) The UV emission, along vertical chords, from an oscillator-initiated plasma. (d) Data from horizontal chords from the same plasma as in (c). The time scales correspond to those in Fig. 2.

Figure 4 (a) Plasma current versus ohmic heating primary current for a cathode-initiated discharge and an oscillator-initiated discharge. Ohmic heating flux is linearly related to the $O H$ primary current. Note that $d I_{p} / d I_{O H}$ is initially larger in the cathode discharge. (b) Plasma current versus total applied energy, from cathode and ohmic heating systems, for the same discharges. The traces have been aligned with zero at the point where the loop voltage is first applied. The extent of the trace to the left of the zero point indicates the amount of energy consumed purely in the preionization and energy to the right of zero is that applied cheiffy by the ohmic heating syrstem (iee text). $d I_{p} / d E_{\text {tot }}$ 
is largest in the initia monents of the osrillator-initiated plasma, but at late times, the $l_{p}$ in the cathode-initiated discharge exceeds that in the oscillator-initiated discharge for the seme input energy: 


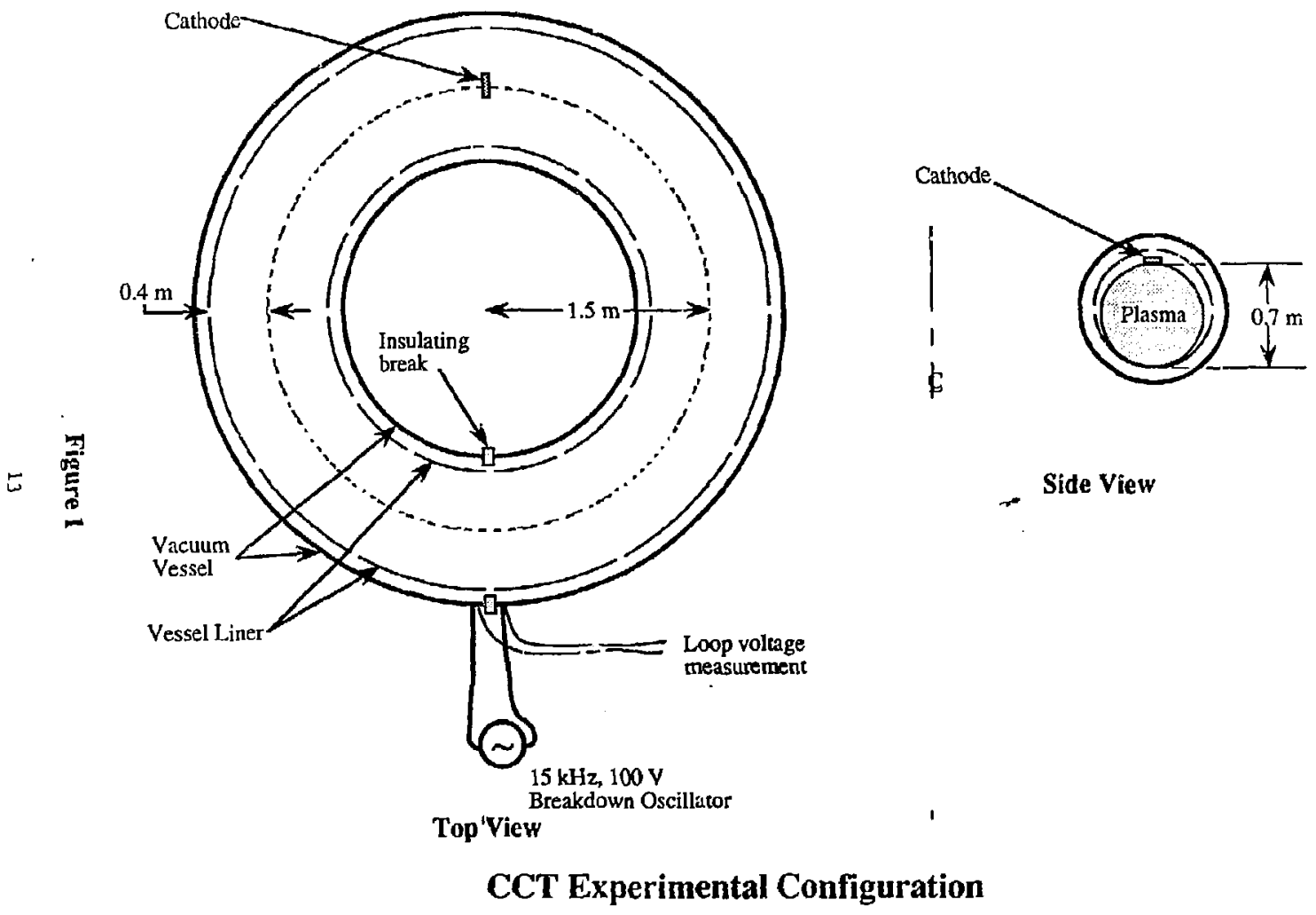




\section{CCT Discharge with Cathode Preionization}

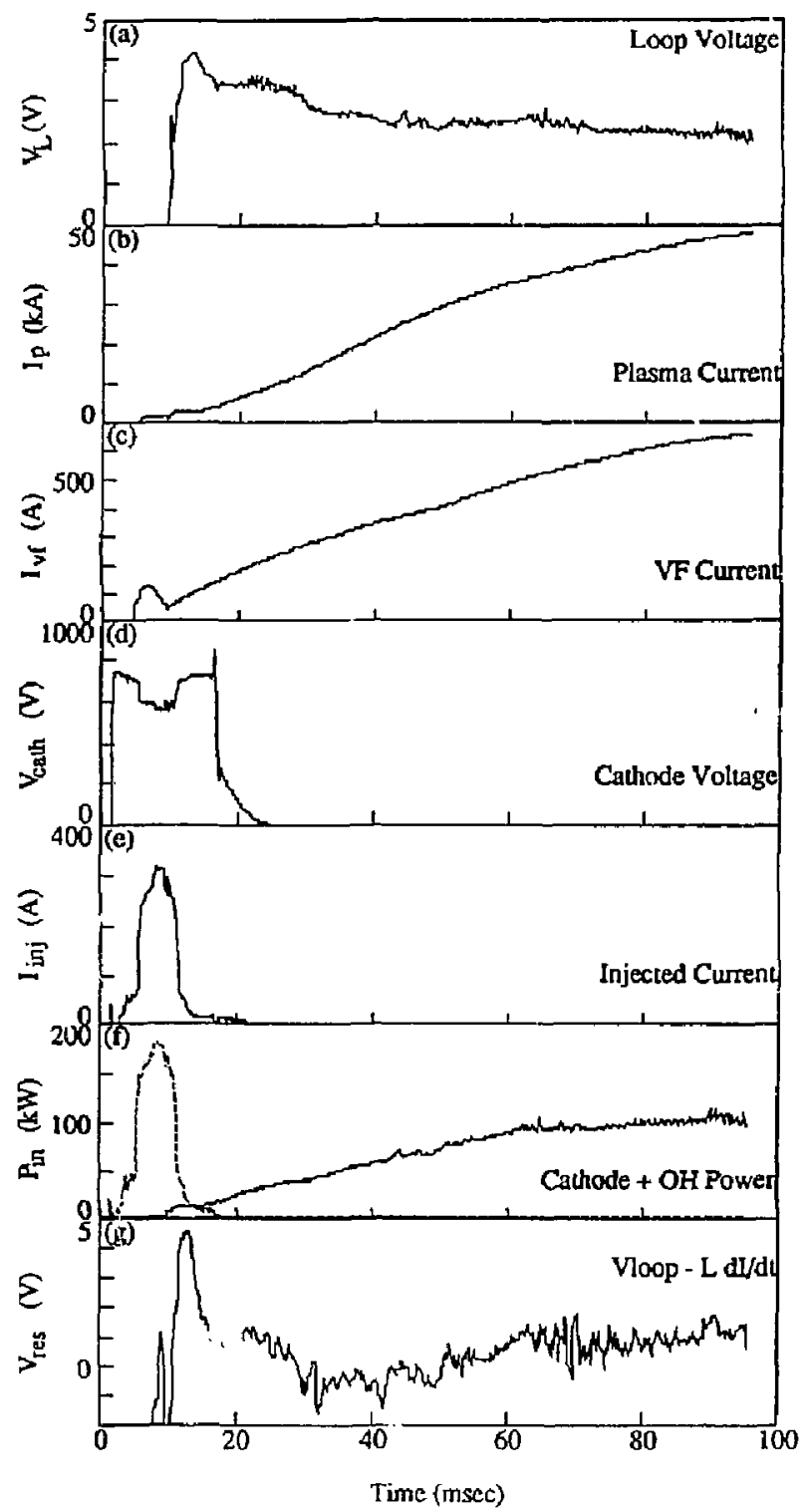

Figure 2(a-g) 


\section{CCT Discharge with $15 \mathrm{kHz}$ Oscillator Preionization}

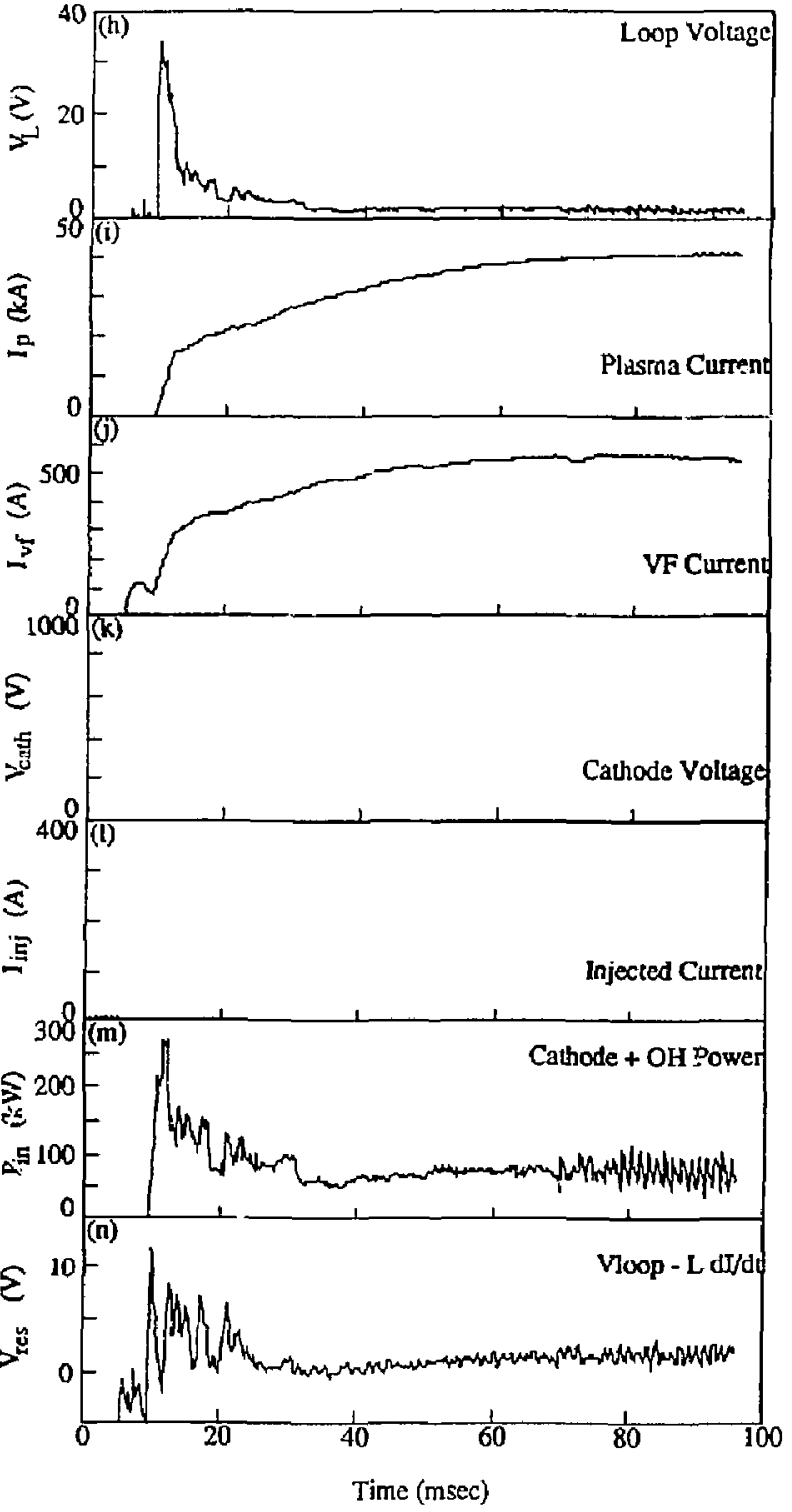

Figure 2(h-n) 


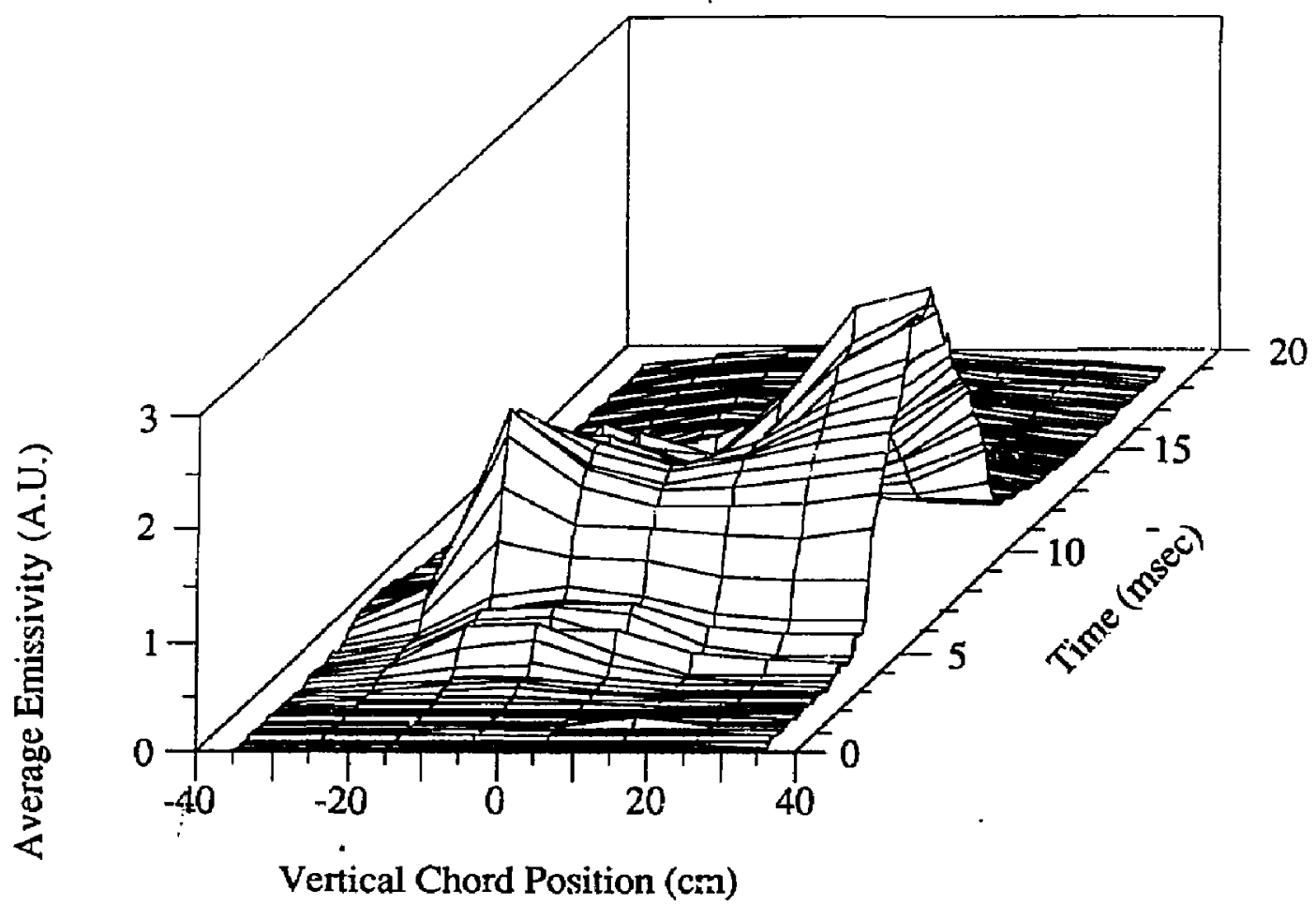

Figure 3a 


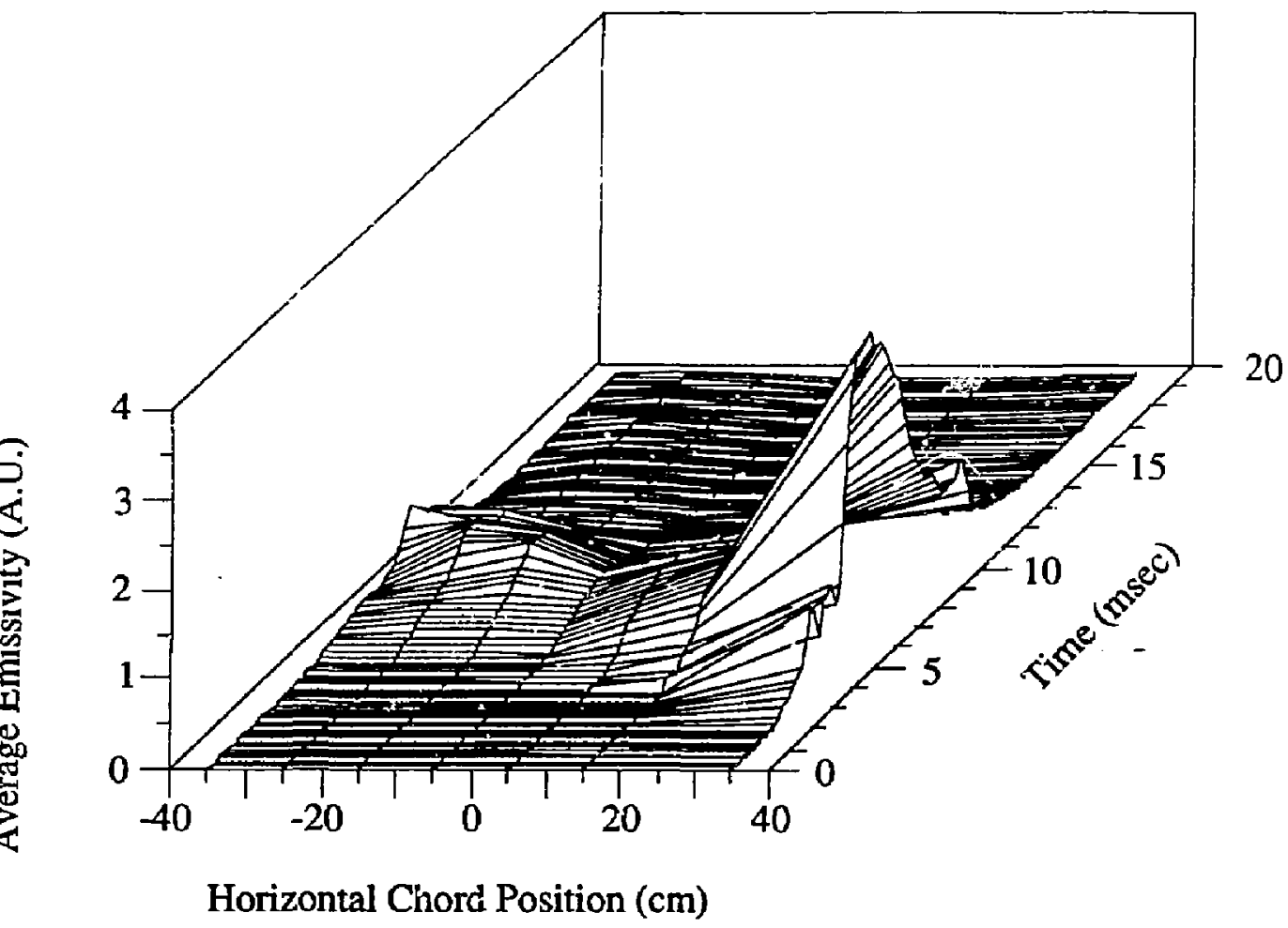

Figure 3b 


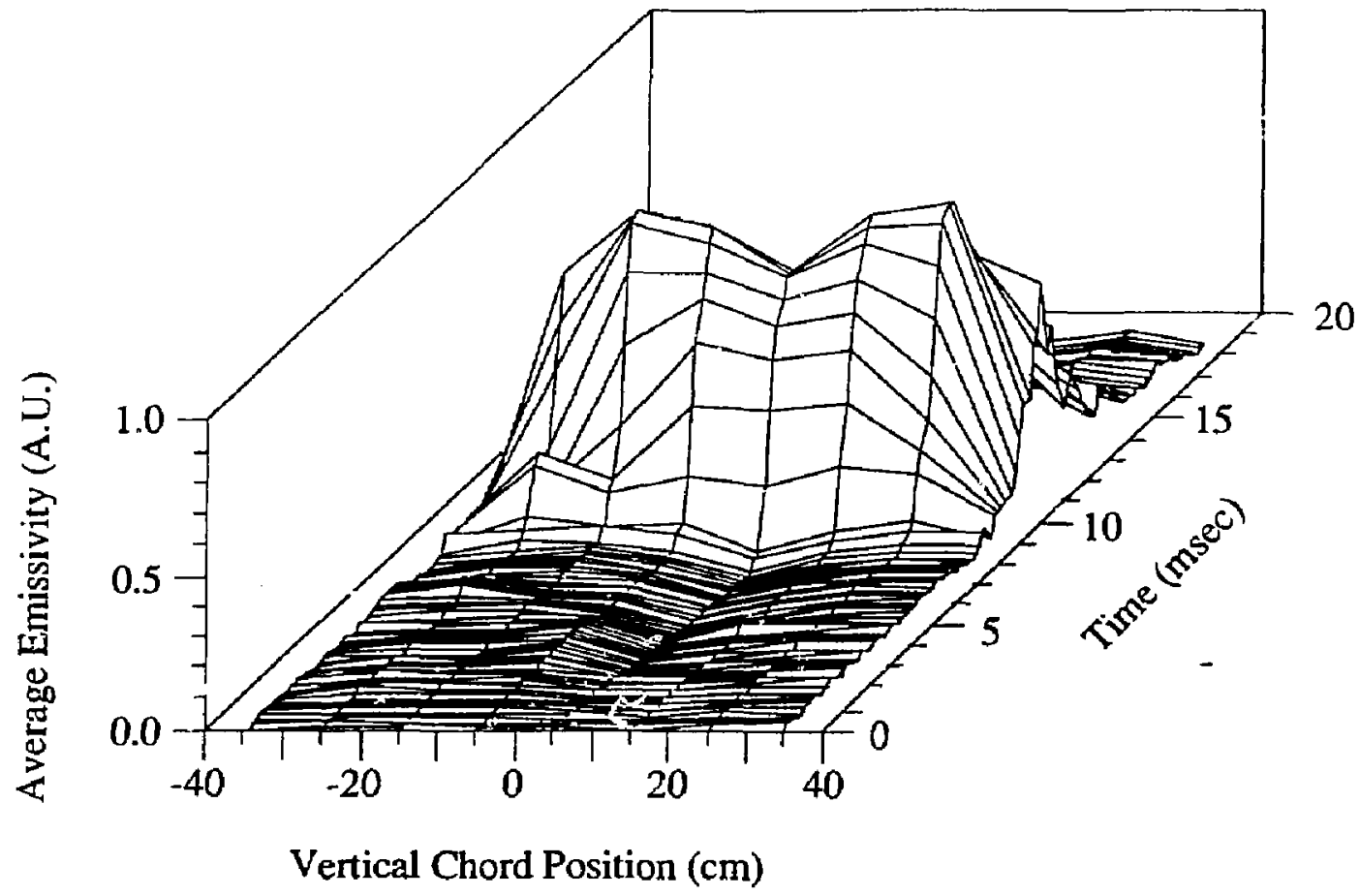

Figure 3c 


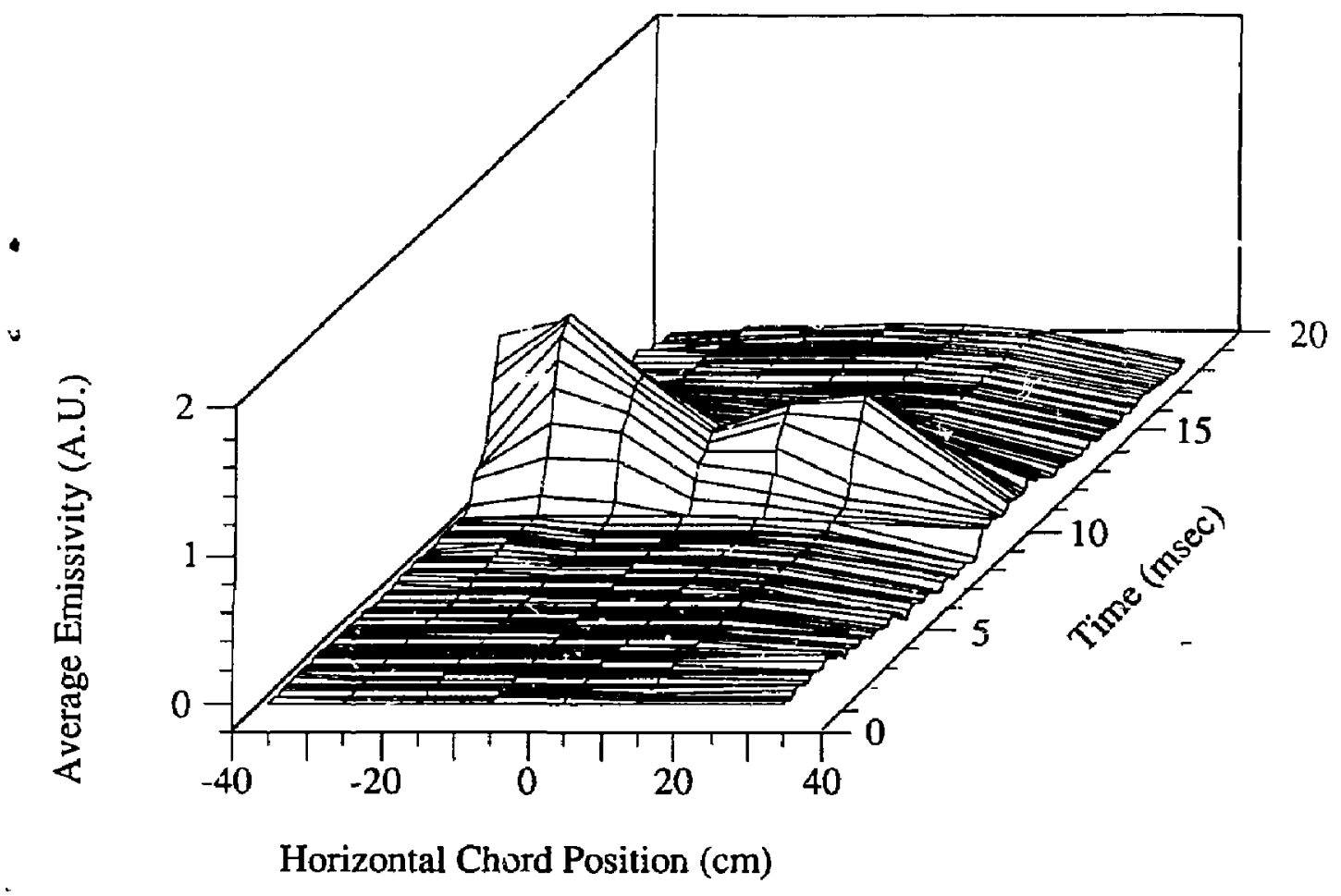

Figure 3d 


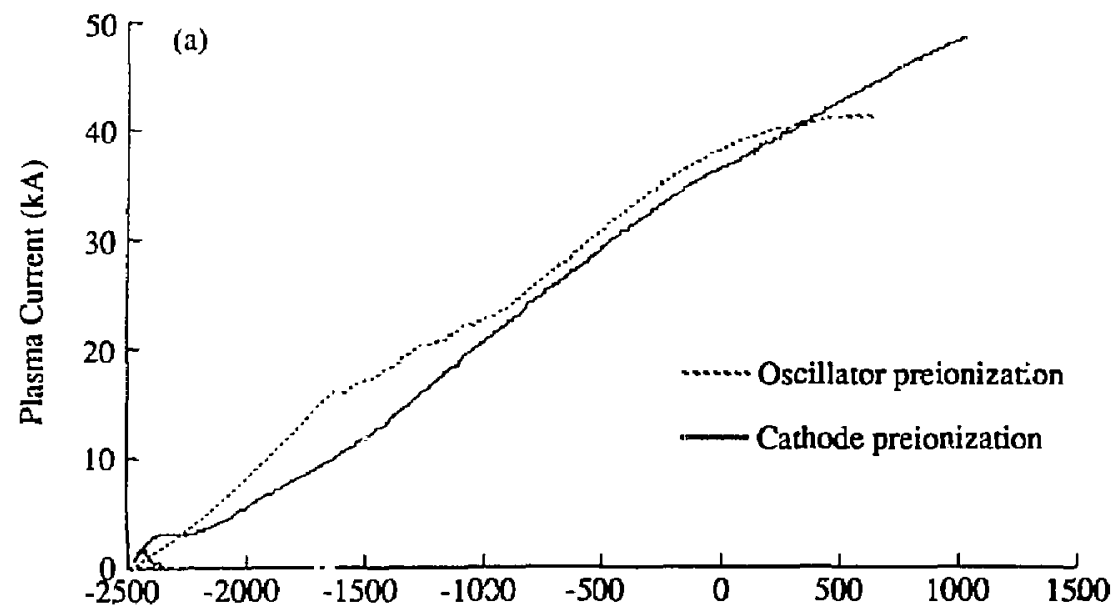

Ohmic Heating Primary Current (A)

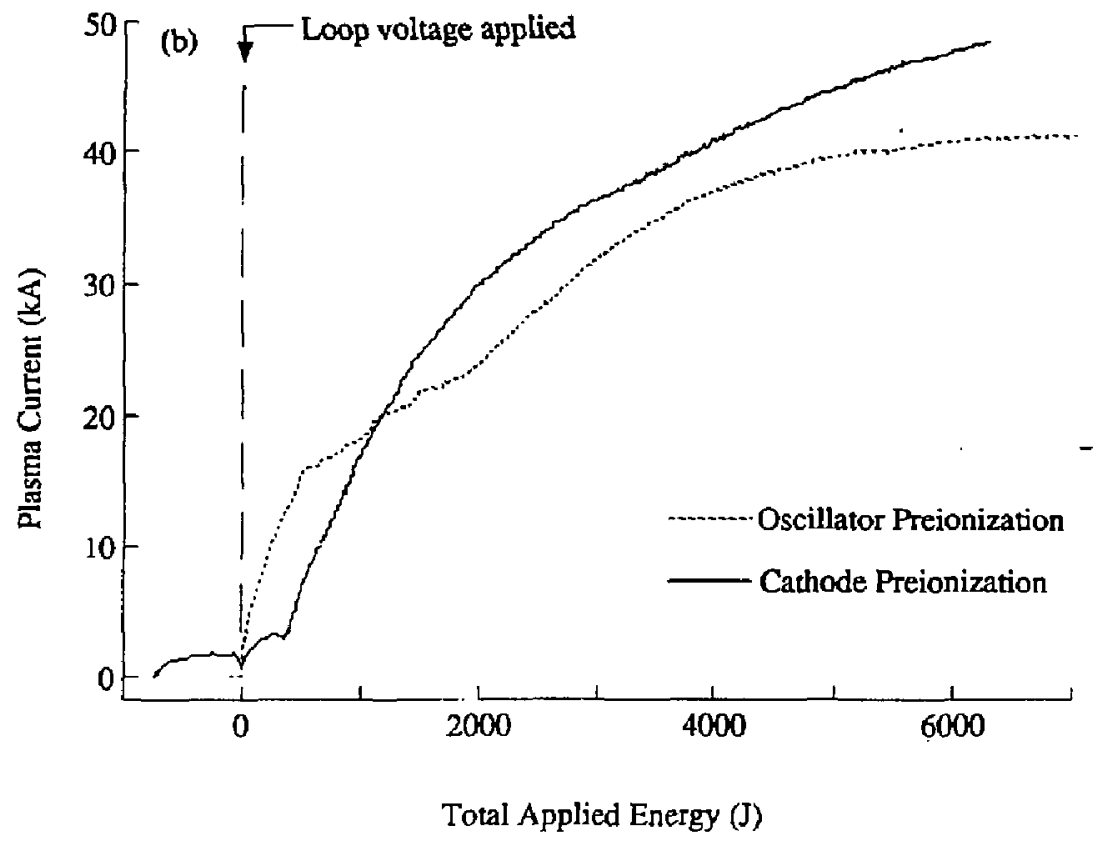

Figure 4 